Musées, Patrimoine et Culture scientifiques et techniques

$117 \mid 2008$

mai - juin 2008

\title{
Web CSTI : le Web est un autre monde !
}

\section{Dominique Boullier}

URL : http://journals.openedition.org/ocim/324

DOI : $10.4000 /$ ocim.324

ISSN : 2108-646X

\section{Éditeur}

OCIM

Édition imprimée

Date de publication : 1 mai 2008

Pagination : 4-13

ISSN : 0994-1908

Référence électronique

Dominique Boullier, «Web CSTI : le Web est un autre monde ! », La Lettre de I'OCIM [En ligne],

117 | 2008, mis en ligne le 09 décembre 2010, consulté le 21 avril 2019. URL : http://

journals.openedition.org/ocim/324; DOI : 10.4000/ocim.324 


\section{Web CSTI :}

\section{le Web est un autre monde !}

\section{Dominique Boullier*}

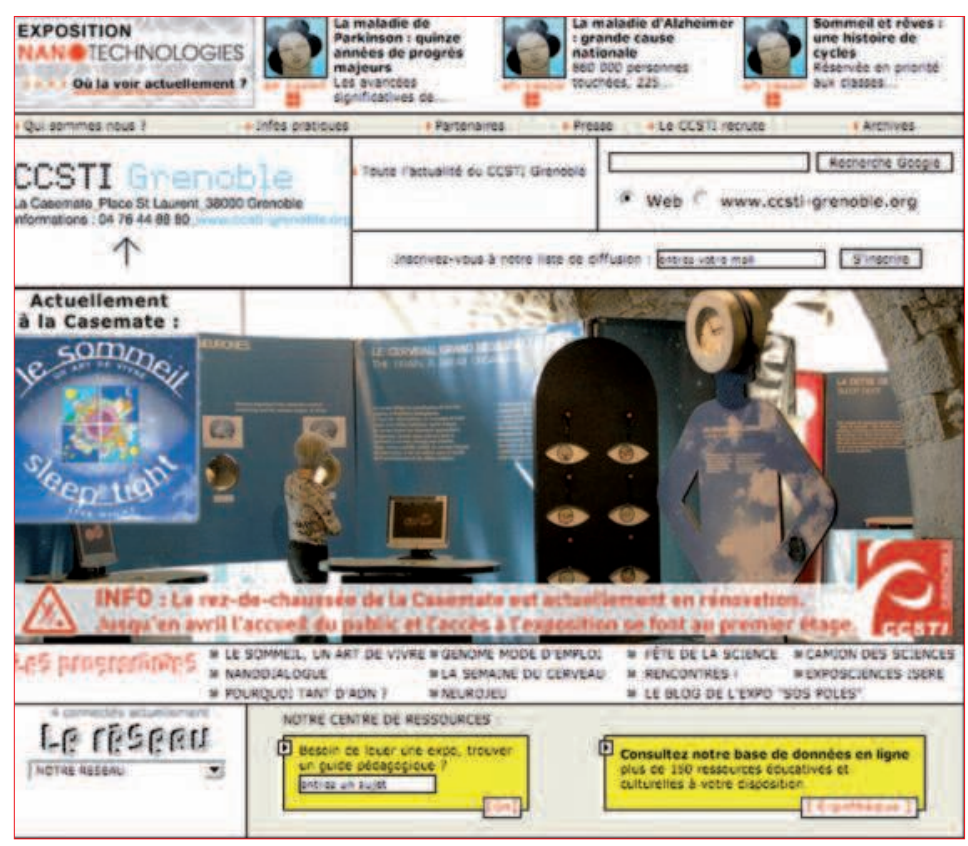

Le CCSTI de Grenoble : un site qui montre la diversité des fonctions d'un CCSTI

* Dominique Boullier est professeur de Sociologie à l'université de Rennes 2 et directeur de l'unité mixte de services CNTRS (UMS 2801) à la Cité des Sciences et de l'Industrie, laboratoire des Usages en Technologies de l'Information numérique dominique.boullier@uhb.fr
L'étude présentée ici - menée à partir d'une sélection de sites Internet les plus représentatifs de la culture scientifique, technique et industrielle - a consisté en une analyse concomitante des pratiques (les usages des internautes) et de l'offre (les stratégies éditoriales des sites) et a permis de définir des stratégies possibles pour la mise en place d'une politique de CSTI sur le Web 2.0.

L'objectif du projet Web CSTI était double ${ }^{(1)}$ : - élaborer des méthodes de compréhension des pratiques des usagers du Web, en combinant des approches diverses, multidisciplinaires, ces méthodes pouvant être utiles à d'autres domaines que la CSTI ;

- proposer une analyse de la dynamique propre au champ de la culture scientifique et technique sur le Web et fournir des résultats sur le domaine et sur certains sites qui soient utiles pour les stratégies éditoriales dans le domaine.

Il ne s'agissait donc pas de proposer des recommandations d'amélioration des " performances » des sites Web de la CSTI, mais de comprendre comment les positions des différents acteurs se reflétaient ou non sur le Web, d'analyser les choix éditoriaux de façon précise et de montrer comment les usagers se repéraient à partir de cette offre. Et surtout, ce travail devait permettre de comprendre désormais à quel point le Web n'est pas un média, n'est pas non plus une vitrine ou un appendice d'une activité réelle, qui lui fournirait toute sa 
matière. Le Web vit de sa propre vie, le Web est un monde à part entière. Notre recherche permet de montrer à quel point il est nécessaire désormais de penser une stratégie Web qui s'appuie sur ces propriétés en lui donnant son autonomie vis-à-vis des équipements, qui pour l'instant subordonnent le Web à leur service.

Notre approche en termes de méthode d'analyse des usages se démarque nettement de plusieurs tendances actuelles.

\section{Une approche originale des usages}

Nous ne pratiquons pas des mesures d'audience. Nous aidons à l'analyse des stratégies éditoriales en permettant de comprendre les écarts éventuels entre des offres et des pratiques. En cela, nous nous démarquons aussi de certaines approches des usages qui considéreraient que les sites ou les pages étudiés sont de purs artefacts sans contexte qui permettent de rendre compte du travail d'interprétation, de navigation et de manipulation des internautes. C'est seulement dans la mesure où nous pouvons rendre compte des cadres et des ressorts qui sont montés conventionnellement dans les constructions mêmes des sites et des pages, de leur contenu, de leur architecture, de leurs formats sémiotiques, que nous pouvons comprendre ce qui fait réagir l'internaute ou lui fait adopter certains comportements lors de nos observations. Nous pensons toujours plus nettement que c'est le couplage offre/usage qu'il faut analyser plutôt que des usages supposés s'imposer sur une offre quelconque ou à l'inverse supposés entièrement subir le formatage de l'offre (2).

\section{Un dispositif de recherche en 4 phases}

C'est pourquoi nous avons monté un dispositif de recherche combinée entre l'analyse de l'offre et les pratiques. Nous y avons ajouté aussi un impératif de variation entre les méthodes, en combinant du quantitatif et du qualitatif. Le programme de travail schématisé comme ci-dessous a été respecté intégralement :

\begin{tabular}{|lll|}
\hline $\begin{array}{l}\text { Plan de travail } \\
\text { Web CSTI }\end{array}$ & $\begin{array}{l}\text { Quantitatif } \\
\text { Automatisation du Recueil } \\
\text { de données en masse }\end{array}$ & $\begin{array}{l}\text { Qualitatif (et quanti) } \\
\text { Sites et pratiques } \\
\text { individuelles }\end{array}$ \\
Offre (les sites) & $\begin{array}{l}\text { 1- Analyse topologique } \\
\text { du Web à partir de } \\
\text { robots }\end{array}$ & $\begin{array}{l}\text { 3- Analyse sémiotique } \\
\text { des sites et découpage } \\
\text { des écrans }\end{array}$ \\
$\begin{array}{l}\text { Demande } \\
\text { (les pratiques } \\
\text { des usagers) }\end{array}$ & $\begin{array}{l}\text { 2- Analyse des parcours } \\
\text { sur sites à partir des }\end{array}$ & $\begin{array}{l}\text { 4- Analyse } \\
\text { oculométrique des } \\
\text { parcours individuels }\end{array}$ \\
\hline
\end{tabular}

\section{Méthodes}

L'AMCSTI a proposé une sélection d'un corpus de sites Internet parmi les plus représentatifs des différentes familles d'institutions réparties sur tout le territoire. Une exploration de ces 31 sites par robots logiciels, méthode dite de " crawling » (méthode RTGICostech-UTC) a permis d'établir des agrégats autour des liens hypertextes. Dix sites ont été retenus :

1. Cité des Sciences et de l'Industrie

(www.cite-sciences.fr)

2. Musée des Arts et Métiers

(www.arts-et-metiers.net)

3. Onera, office national d'études et de recherches spatiales (www.onera.fr)

4. Les Petits Débrouillards

(www.lespetitsdebrouillards.org)

5. Cap Sciences (www.cap-sciences.net)

6. CCSTI de Grenoble (www.ccsti-grenoble.org)

7. Parc naturel régional du Lubéron

(www.parcduluberon.com)

8. Écomusée du pays de Rennes

(www.ecomusee-rennes-metropole.fr)

9. Aquariums de France

(http://membres.lycos.fr/aquafrce)

10. Muséum d'Histoire naturelle de Bourges

(www.museum-bourges.net)

Pour diverses raisons techniques, de disponibilité de données ou de contact difficile avec l'institution, certaines phases de l'étude n'ont pu être réalisées avec tous les sites (3). Notre étude n'avait pas de prétention de représentativité « stricte », elle s'est assurée cependant d'une représentativité correcte des types de site, de la faisabilité des méthodes mises en œuvre pour ces sites et d'une aide réelle à la réflexion stratégique sur les choix éditoriaux de la CSTI en général.

\section{Analyse automatisée des parcours en masse}

Un inventaire des méthodes existantes pour l'analyse des parcours en masse (et non des parcours individuels) a été conduit ainsi qu'une critique des limites des systèmes experts d'une part et des méthodes par classification d'autre part. Un algorithme de classification spécifique a été développé dans le cadre du LIP6 (Leader Ant) pour réaliser une partition des sessions de navigation des internautes en groupes homogènes appelés clusters. Ces sessions ont été récupérées à partir des données log fournies par les serveurs. 
L'exploitation des données de base a nécessité un lourd travail de nettoyage de ces données sachant que plus de $50 \%$ des logs de trafic sur tous les sites tracent en fait le passage des robots (des moteurs de recherche notamment). Le site de la Cité des Sciences et de l'Industrie n'a pu être exploité en raison d'une erreur de fichier source. Il ne s'agissait pas de fournir une mesure d'audience comme peut le faire Google Analytics qui agrège toutes les données mais ne permet pas de comprendre les logiques internes des parcours individuels, que l'on doit, pour ce traitement de masse, regrouper en profils de navigation grâce à l'algorithme de classification développé. Les accès directs à certaines pages des sites (comme il est souvent possible à partir des moteurs de recherche) n'ont pas été pris en compte puisque l'on s'intéresse à des parcours à partir de la page d'accueil.

Les données produites comportent le nombre de navigations et de sessions uniques par site, la longueur des sessions, et, pour chaque profil, le top 20 des pages les plus fréquentées.

Les pages comportant les informations pratiques ainsi que celles proposant des téléchargements sont parmi les plus visitées systématiquement et organisent des profils de parcours centrés sur ces activités. Les expositions constituent un autre profil de navigation (que l'on trouve par exemple à Cap Sciences et au CCSTI Grenoble), ainsi que les départements de recherche (Onera) et les recrutements (Onera).

Ce type d'approche permet de mettre en évidence à quel point les consultations comportent des visées différentes et doivent être distinguées dans les analyses : chercher les informations pratiques pour venir

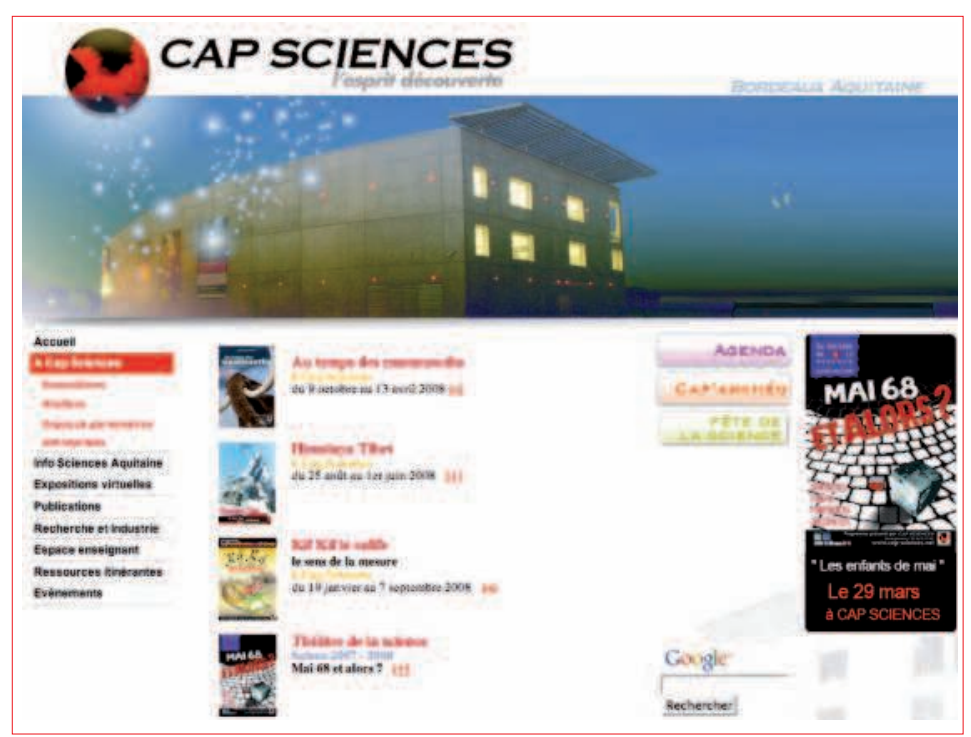

Cap Sciences : un site agrégat visiter tel écomusée est finalement éloigné d’une recherche d'information à partir des documents associés à une exposition particulière. La capacité à identifier ces profils type de navigation, en mesurant leur poids respectif, va au-delà d'une simple mesure d'audience et permet de modifier les stratégies éditoriales en tenant compte de logiques utilisateurs de parcours et non plus seulement de pages vues.

\section{Analyse sémiotique des sites}

La méthode nécessitait de comprendre les processus de reconnaissance de formes (Gelstalt) : comment un internaute peut-il produire des attentes cohérentes à partir des premiers indices qu'il perçoit sur un site ? Comment les concepteurs de site peuventils s'appuyer sur ces supposées attentes, en tenant compte des cultures de leurs futurs visiteurs ? Cela les conduit à s'appuyer sur des «traditions » (Manovich), des conventions (Thévenot) ou des genres (Genette). Pour l'analyse, il nous faut produire les vocabulaires de description pertinents qui permettraient de rendre compte de ces conventions. Or, le Web, par définition quasiment, est instable, émergent, et capable de combiner plusieurs traditions ou conventions.

L'évaluation a donc porté sur les saillances (ce qui attire l'attention) qui sont perçues par les internautes et qui leur permettent de cadrer leur expérience comme " un genre de ", sans que cette convention soit nécessairement reconnue ou officialisée. Par exemple, le facteur position dans la page : le lecteur du support papier, par la forme même du dispositif du journal, porte son regard d'abord sur le quadrant haut supérieur et les traditions de l'imprimé ont formé tous les professionnels à exploiter cette propriété. Or, celle-ci ne fonctionne pas pour les écrans puisque l'attention est d'abord focalisée sur le centre. La tradition de l'imprimé ne peut donc plus fonctionner.

Les saillances ont la capacité d'attirer l'attention (dans ses deux valeurs : intensité + durée) et l'analyse des sites Web doit permettre de rendre compte de cette capacité d'attraction de l'attention. Mais elle est toujours dépendante du contexte et ne peut être ramenée à des règles de genres définies a priori ou à des recettes comme le font croire beaucoup de manuels du type « how to ». C'est pourquoi, il a été indispensable de faire appel à des jurys qui vont collectivement produire leur évaluation de ce qui attire leur attention et pour identifier les types de saillances dominantes. À ce moment, nous pouvons mieux comprendre comment les sites Web sont toujours des compositions entre plusieurs traditions. 
La méthode repose sur plusieurs sources :

- une analyse de l'expert,

- un entretien avec l'éditeur (pour repérer ses intentions éditoriales),

- des jurys d'internautes (hors action, c'est-à-dire seulement en examinant les pages d'accueil). Le fait de centrer sur les pages d'accueil conduit à ne traiter que $25 \%$ du trafic, rappelons-le. Mais les attentes (ou présomptions d'isotopie) sont construites à ce moment.

Les analyses ont porté sur 4 sites en particulier et permettent de bien différencier des stratégies sémiotiques particulières et d'observer comment elles sont perçues par les utilisateurs :

- la Cité des Sciences et de l'Industrie présente toutes les contraintes d'un portail. De ce fait elle doit gérer une hétérogénéité sémiotique sur sa page d'accueil, qui ne produit pas des attentes reconnues dans la navigation par la suite. Deux formats de présentation de toute l'offre existent d'ailleurs, avec une page reprenant la convention de la mosaïque sur la télévision. Les innovations interactives qui sont une ressource originale sont plutôt masquées à l'intérieur des sous sites par exemple. Nous verrons que tout cela a des incidences non négligeables sur la rapidité de repérage lors des navigations ;

- le site du musée des Arts et Métiers a fait un choix de convention forte, celui du " CD-ROM culturel », avec choix d'un format écran et non page, maintenu en permanence. Les interfaces sont interactives à la mode d'un CD-ROM et le visuel très présent (mais il se limite à une incitation à la visite et ne peut pas s'y substituer);

- l'Onera adopte une convention de site de presse, avec la dépêche comme format type central, mais quelque peu détourné puisqu'elles sont encadrées. Les photographies de qualité qui sont présentes

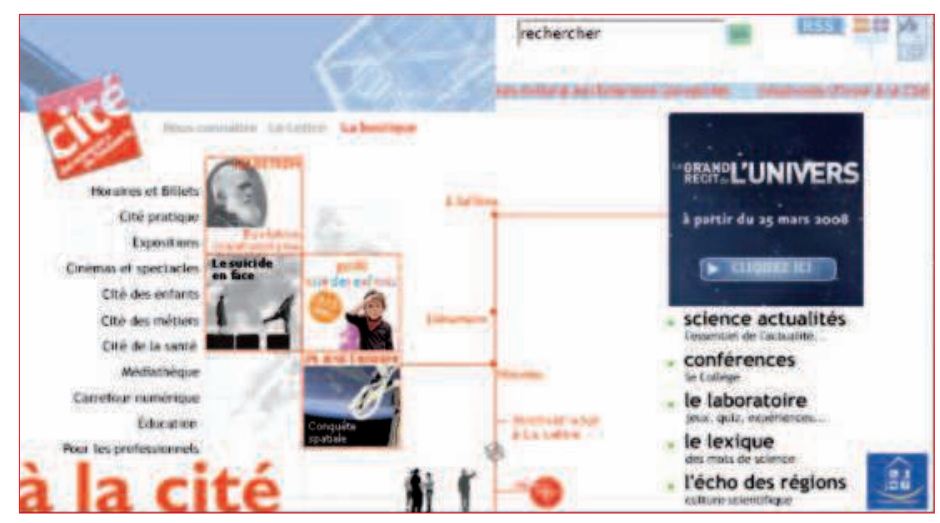

La Cité des Sciences et de I'Industrie : un site portail orientent alors vers une convention de magazine haut de gamme, de didactique des sciences car les photographies sont toujours accompagnées de très longs commentaires ;

- Cap Sciences adopte un modèle agrégat typique de la diversité des fonctions d'un CCSTI (voir aussi Grenoble dans notre échantillon), qui n'est pas un portail car la profondeur est ensuite faible. Les animations des titres ne semblent guère apporter autre chose que du bruit. La multiplication des catégories en entrée et leur faible structuration se révélera un avantage pour les utilisateurs comme le montrera l'étude oculométrique.

\section{Analyse des parcours oculométriques}

La recherche a fait l'état de la littérature sur les analyses oculométriques des sites Web. Le protocole a été construit uniquement autour de la recherche d'informations, cette précision est importante à prendre en compte car d'autres activités sont réalisées sur les sites. 27 participants ont réalisé 3 tâches de difficultés différentes demandant un, deux ou trois clics pour obtenir l'information correcte. Les mouvements oculaires analysés avec le système «Tobii » étaient le nombre de fixations et la durée de fixation. L'échec ou la réussite étaient aussi utilisés comme indicateurs. Enfin un questionnaire de satisfaction était administré.

Trois groupes de sites sont apparus selon la difficulté des internautes. Certains sites peuvent être plus difficiles parce qu'on ne sait pas où trouver l'information (mais on peut cliquer pour chercher) mais d'autres parce qu'on ne voit pas où la chercher (on est paralysé). Les sites du muséum de Bourges, du CCSTI Grenoble, de Cap Sciences et du parc du Lubéron sont apparus les plus faciles. La variable âge était analysée de façon particulière et cela a permis de confirmer les difficultés plus grandes éprouvées par les personnes âgées.

Les fixations oculaires sont apparues plus nombreuses sur les sites du groupe facile (avec réussite et rapidité de succès), à la fois avant de cliquer dans toutes les pages mais spécifiquement sur les pages d'accueil. Ce qui peut paraitre contre intuitif puisqu'on aurait pu supposer qu'un grand nombre de fixations prend plus de temps ou manifeste une plus grande désorientation. De plus les notes de satisfaction étaient plus élevées pour ces sites.

Pour analyser ces résultats et comprendre leur caractère non prévisible, il a été nécessaire de décrire les propriétés sémantiques des sites et de l'offre de liens notamment. Il apparaît ainsi que les sites portails 
qui comportent des sous sites ont plus de mal à offrir des accès multiples et simples à partir de catégories de base : ils doivent fonctionner avec des méta catégories, nécessairement trop larges ou impropres. Les utilisateurs passent cependant vite sur la page d'accueil mais se retrouvent souvent dans une impasse lorsqu'ils constatent que la méta catégorie qu'ils avaient suivie ne comprend pas l'objet de leur recherche. Alors que pour les sites peu profonds qui offrent une grande quantité de liens sur la première page - liens non structurés par méta catégories - il est certes nécessaire de passer plus de temps à scanner toutes les possibilités (d'où le nombre de fixations élevé) mais ensuite, les chances d'avoir été bien aiguillé sont plus grandes.

Enfin, une analyse par graphe contextuel a permis de comparer les parcours effectués aux parcours possibles et de vérifier l'influence du contexte sur les choix de l'utilisateur.

\section{Analyse du champ de la CSTI}

Ces analyses sur les consultations et les pratiques des internautes doivent cependant être mises en perspective par rapport à l'offre dans son ensemble, qui peut constituer un " champ ", pour reprendre le concept de Pierre Bourdieu. Le champ de la diffusion de la culture scientifique et technique repose en France sur 174 musées scientifiques, 54 structures associatives et CCSTI, 13 associations d'éducation populaire regroupées au sein du CIRASTI (collectif interassociatif

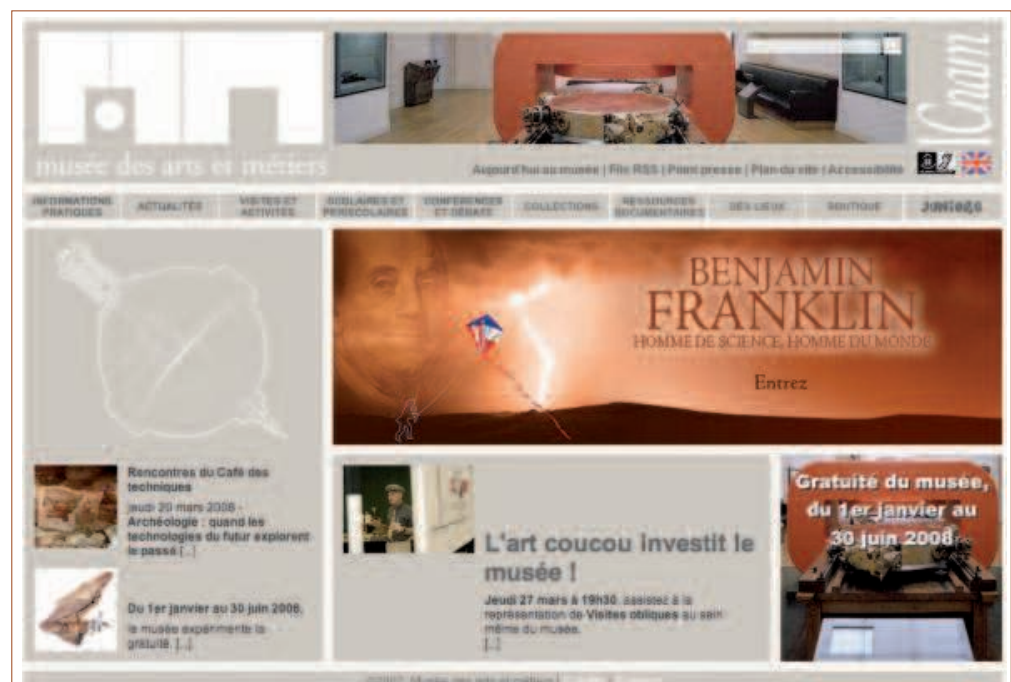

Le musée des Arts et Métiers : un CD-ROM culturel au format écran pour la réalisation d'activités scientifiques et techniques internationales) et sur environ 150 autres associations.

Malgré le caractère quasi évident d'une offre Web en provenance de ce champ, il n'est pas certain que, malgré les bonnes intentions et les recherches d'innovation, l'offre des institutions de diffusion de la CSTI sur le Web soit adaptée aussi bien aux publics déjà conquis comme aux publics à conquérir. Il ne s'agit pas seulement de décrire des publics mais bien de mettre en évidence les stratégies possibles sur le Web. Nous nous situons de ce fait dans la lignée des recherches conduites en Lorraine par Marie-Jeanne Choffel-Mailfert ${ }^{(4)}$ sur la CSTI régionale lorraine. Ses travaux sont parmi les rares qui permettent à la fois d'avoir une observation fine des dispositifs mis en place et de proposer une analyse des stratégies possibles selon les types d'acteurs.

\section{La méthode $\mathrm{d}^{\prime}$ analyse topologique des sites Web de la CSTI (5)}

Ce travail s'appuie sur une version élargie de la théorie des agrégats. En effet, la théorie « classique » des agrégats (Kleinberg) ne permet de décrire comme « agrégat » qu'un ensemble de sites organisés en loi de puissance, alors même que nous savons que certains domaines sont très structurés mais d'une façon différente. Nous avons donc élargi la notion « d'agrégat documentaire Web » à d'autres formes de structures, selon deux critères : existence ou non d'un cœur ou centre, existence ou non d'une trame maillée.

Les outils dont le groupe RTGI dispose pour réaliser cette étude sont de deux types : les outils d'extraction et d'indexation des données issues du Web, et les outils de traitement et d'analyse de ces données. Nous utilisons de petits robots semblables à ceux des moteurs de recherche pour parcourir le Web automatiquement. Ces robots logiciels, appelés « crawlers », rapatrient les données disponibles sur le Web dans une base de données. Classiquement, les crawlers parcourent les pages de lien en lien à partir d'un ensemble de pages données appelées «points d'entrée » et jusqu'à un nombre de liens hypertextes fixé à l'avance, appelé "profondeur ». L'étape de rapatriement des données, appelée « crawl », s'arrête lorsque toutes les pages ont été visitées jusquà la profondeur paramétrée. Toutefois, dans notre cas le fonctionnement des robots est sensiblement différent. En effet, nous nous intéressons aux sites et non aux pages. Par conséquent, les robots sont paramétrés de telle sorte quilis parcourent les sites à partir de leur page d'accueil, jusquà une profondeur en pages fixée, en 
l'occurrence 3. En outre, nous n'utilisons pas de profondeur en sites pour stopper le « crawl » : les robots hiérarchisent les sites à indexer en fonction de leur connectivité, et s'orientent naturellement vers les sites les plus connectés à des fins d'optimisation du processus. Ainsi, le « crawl » s'arrête lorsque le nombre de sites indexés est jugé suffisant.

Une fois le « crawl » arrêté, une étape de finalisation permet d'exploiter la base de données. Les données utiles sont compilées sous la forme d'un ou plusieurs fichiers compatibles avec les outils d'analyse nécessaires à l'étude. Dans notre cas, les « crawls » prennent la forme d'un graphe enrichi : les sites sont représentés par des nœuds et les liens hypertextes par des graphes, et diverses données sont attachées à ces éléments pour en permettre l'analyse. Notre principal outil d'analyse est un logiciel de traitement des graphes (GUESS) que nous avons modifié selon nos besoins.
Le tableau suivant représente les meilleures autorités et les meilleurs « hubs ». Un score d'autorité est élevé lorsqu'un grand nombre d'autres sites pointent vers lui avec des liens hypertextes : on considère qu'il fait référence pour tout un agrégat, pour tout un domaine (ici le CNRS par exemple, puis la Cité des Sciences). Un score de " hub » est élevé lorsqu'un site pointe vers beaucoup d'autres sites et constitue ainsi un point de passage obligé, non plus une référence mais une porte d'entrée vers tous les sites importants d'un domaine (par analogie avec un « hub» dans la navigation aérienne). On peut voir qu'il y a très peu de sites à haut score (de l'un ou de l'autre) et beaucoup de sites à faible score. Nous sommes dans un réseau dit « à invariance d'échelle ", avec très peu de sites très connectés et beaucoup de sites peu connectés. Les courbes d'autorité et de « hub » ont bien la forme d'une « loi de puissance », même si on peut observer un petit décrochage dans la courbe des « hubs ».

\section{Liste des sites appartenant au coeur, par score d'Autorité décroissant :}

\section{0,0523}

0.0523

www.planetenature.erg

www.ctba.fr

www.latecoere,com

0,0479 perso.wanadoo.frmainesciences

$0,0469 \quad$ www.museum.nantes.fr

$0.0468 \quad$ www.cesticerse.assa.fr

$0,0463 \quad$ www.scienceanimation.org

0.0455

0,045

0,044

0,0439

0,0436

0,0433

0,0429

0,0429

0,042

0,0423

0,042

0,0419

0,0416

0,041

0,0414

0,0405

0,038 ?

0,0382

0,0363

0,0362

0,035

0,0350

0,0349

0,0344

\section{www.ecean.asso.fr}

www.mollat.cem

www.quaternet.fr

www.agrotecfrance.com

www.adera.fr

www.cestib.org

www.sciencespoberdeaux.fr

www.bordeauxunitec.com

www.technowest.com

www.crrhonealpes.fr

www.agropole.com

www.itergeom

www.iftssls.com

membres.lyces.frecstidelaval

www.cyberpapy.cem

www.franceinfo.com

www.univmontp2.fr

www.cirasti.erg

www-apesa.asso.fr

www.caminteresse.fr

www.edf.fr

\section{Liste des sites appartenant au coeur, par score de Hub décroissant :}

www.museumlyon.org

perso.wanadoo.frccstilacgedyssee

www.parcsnaturelsregionaux.tm.fr

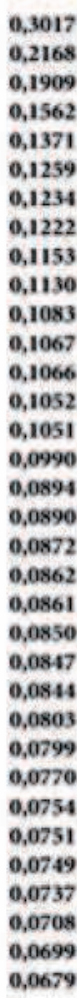

\begin{tabular}{|c|c|c|}
\hline nww.capsciences.net & 0,0435 & www.agropole.com \\
\hline www.citesciences.fr & 0,0429 & www.infoscience.fr \\
\hline www.cnrs.fr & 0,0420 & www.technowest.com \\
\hline www.ecim.fr & 0,0414 & www.edf.fr \\
\hline www.ubordeaux1.fr & 0,0392 & www.reservesnaturelles.org \\
\hline www.planetenature.org & 0,0384 & www.nouvelobs.com \\
\hline www.bordeauxunitec.com & 0,0358 & www.agorasciences.org \\
\hline www.ubordeaux3.fr & 0,0349 & www.crrhonealpes.fr \\
\hline www.scienceanimation.erg & 0,0347 & www.physique2005.org \\
\hline perso.wanadoo.frassoc.lorient.cesti & 0,0345 & www.enslyon.fr \\
\hline perse.wanadoe.frecstilacqedyssee & 0,0343 & www.ccstib.org \\
\hline www.enera.fr & 0,0342 & www.iledefrance.fr \\
\hline www.sciencespobordeaux.fr & 0,0342 & www.univpau.fr \\
\hline www.labri.fr & 0,0292 & www.adera.fr \\
\hline www.larecherche.fr & 0,0235 & www.apesa.asso.fr \\
\hline www.inrp.fr & 0,0217 & www.enscpb.fr \\
\hline www.citedelamer.com & 0,0140 & www.cirasti.erg \\
\hline www.cnes.fr & 0,0119 & www.systonic.fr \\
\hline www.estia.fr & 0,0094 & www.mollat.com \\
\hline www.pasteur.fr & 0,0085 & www.neufplanetes.org \\
\hline www.ademe.fr & 0,0085 & www-artsetmetiers.net \\
\hline www.uberdeaux 2.fr & 0,0082 & www.cybersciences.com \\
\hline www.cnam.fr & 0,0079 & www.cyberpapy.com \\
\hline www.univmontp2.fr & 0,0054 & www.franceinfo.cem \\
\hline membres.lycos.frcestidelaval & 0,0048 & quinzaine.equitable.free.fr \\
\hline www.csstigrenoble.erg & 0,0038 & www.ecean.asso.fr \\
\hline www.francescience.erg & 0,0033 & www.ecoledesavignac.com \\
\hline www.momes.net & 0,0031 & www.enseirb.fr \\
\hline www.ouestfrance.fr & 0,0024 & www-agrotecfrance.com \\
\hline www.aquitaine.fr & 0,0016 & www.ensam.fr \\
\hline www.relaisdsciences.org & 0,0000 & www.talenceinnovation.fr \\
\hline www.acbordeaux.fr & 0,0000 & www.amazon.fr \\
\hline ww.pourlascience.com & 0,0000 & www.cieletespace.fr \\
\hline www.radiofrance.fr & 0,0000 & www.lescale.net \\
\hline
\end{tabular}




\section{Les limites de la CSTI instituée}

L'offre telle qu'elle est structurée et révélée par nos analyses topologiques du groupe RTGI présente un contraste saisissant.

Les sites Web de quatre des institutions majeures de la CSTI (www.cite-sciences.fr, www.arts-et-metiers.net, www.palais-decouverte.fr, www.mnhn.fr) sont bel et bien au cœur du domaine. Ils ne sont certes pas les seuls à bénéficier d'une large audience sur le Web, mais aucun autre site parfaitement centré sur la CSTI ne peut les concurrencer.

Chacun des quatre sites a une forte connectivité. En outre, ils sont connectés presque exclusivement avec d'autres sites du cœur de la CSTI sur le Web : des CCSTI, des sites d'universités ou de grandes institutions... Dans leur voisinage immédiat on peut toutefois observer des aquariums, des parcs naturels et des muséums : ce sont pour la plupart des sites qui les référencent, mais qui ne sont pas référencés en retour.

Les CCSTI sont bien le relais de la structuration générale du domaine en France. Ils sont en effet bien reliés aux grandes institutions centrales et ils ont l'avantage d'organiser le domaine à un niveau local. Mais ce label CSTI ne permet pas aux CCSTI de prétendre regrouper toutes les activités locales, ou leur servir de portail. Nous constatons au contraire que les autres institutions qui travaillent dans le champ sont finalement peu nombreuses à revendiquer l'appellation et, plus important, à offrir des connexions au reste du domaine. L'émiettement du champ est assez net de ce point de vue et rend difficile toute visée stratégique réelle. Le rôle des associations semble de ce point de vue assez faible en dehors de la dimension non négligeable d'échange d'expériences.

La topologie du domaine CSTI en France peut être finalement présentée de la façon suivante, une fois éliminé tout le détail technique des résultats.

\section{Une CSTI coupée des amateurs de sciences?}

Dans un domaine comme celui des aquariums, les frontières avec l'activité ordinaire du Web et par hypothèse avec toute l'activité liée à des pratiques scientifiques ordinaires ou populaires, semblent bien étanches. Les aquariums ont une politique Web centrée sur leur équipement, dans ses dimensions d'équipement touristique local, le site Web devant servir à drainer des visites avant tout. La valeur ajoutée des sites est à peu près nulle en termes d'information ou de ressources d'information. Leur faible connectivité apparaît vis-à-vis de toute la culture scientifique et technique instituée, mais aussi entre eux, comme si leur logique d'équipement en faisait plus des concurrents sur le marché touristique que des partenaires. Plus étonnant mais plus



Panorama de la culture scientifique, technique et industrielle en France 
intéressant aussi, c'est en fait un site indépendant qui a fini par constituer la meilleure connectivité et à devenir non seulement un « hub » mais aussi une autorité : ce portail de fait, entièrement tenu par des amateurs passionnés, est capable de référencer tout ce qui peut se rapporter aux aquariums dans le monde entier (connexion mondiale qui est une exception dans les sites Web de la CSTI française) tout en prenant en compte toutes les activités plus populaires d'aquariophilie les plus ordinaires, avec les sites pratiques pour entretenir son aquarium, pour le peupler, pour acheter du matériel...

Ce cas est unique dans notre corpus mais cela ne lui enlève pas sa valeur exemplaire. Telle qu'elle apparaît sur les sites Web, la CSTI française s'est définie dans une coupure à peu près complète avec les " amateurs de science » (terme employé par B. Latour). Amateurs qui sont aussi praticiens et qui l'abordent par des activités proches du bricolage, du hobby, de la passion, mais qui pourtant constituent le ferment d'une culture scientifique et technique vivante et réellement partagée au point de devenir ce qu'on appelle désormais sur le Web 2.0, des " proams » (professionnels amateurs). La CSTI, malgré (ou à cause) de son penchant diffusionniste, semblerait ignorer tout ce qui se fait en dehors d'elle, si on la regarde à travers le Web.

\section{Avantages et risques de la focalisation sur un équipement local}

Le deuxième constat clairement apparu à travers l'analyse de la topologie du Web de la CSTI porte sur une certaine tension entre insertion locale et vocation nationale, ou plutôt sur la forte influence d'un équipement nécessairement situé quelque part (!) sur sa politique Web qui pourrait théoriquement en être indépendante. Ainsi nous observons ce centrage sur l'équipement dans le cas des aquariums mais aussi, et de façon presque caricaturale, dans le cas des planétariums. Toute l'information qu'ils diffusent est en fait centrée sur leur activité interne en termes d'horaires et d'animation, avec quelques images peu nombreuses pour attirer le client pourrait-on dire. Or, on peut très bien comprendre les contraintes et les stratégies qui les conduisent à ce type d'approche. Leur équipement souvent prestigieux, qui est un atout, peut en effet devenir un frein ou tout au moins un cadre très contraignant. La fréquentation de leur établissement, peut-on supposer, est le critère décisif de leur évaluation par les différents bailleurs de fond. Ce cas de figure des aquariums ou des planétariums n'est pas isolé, on le trouve explicitement pour certains écomusées, mais plus généralement, c'est un enjeu pour tous les équipements de la CSTI. Leur insertion locale fait leur différence par rapport aux institutions générales ou à vocation nationale. De ce fait, certaines des missions ou plutôt des opportunités offertes par le Web ne sont pas prises en charge.

\section{Autres tensions structurantes du champ}

La CSTI comporte aussi une dimension muséale ou plus largement patrimoniale, reposant sur des collections. La plupart des sites n'ont pu, sans doute faute de moyens, que jouer, comme les planétariums, la carte des informations orientées vers la visite de l'équipement et de ses collections, dans un modèle touristique déjà évoqué. Mais certains sites se sont démarqués de cela et ont fait preuve d'ambitions particulières en matière de présentation des collections sur le site Web lui-même (musée des Arts et Métiers), ou encore d'enrichissement de la visite par des informations historiques, scientifiques, animées (Agropolis).

La CSTI est supposée faire partager l'activité de base des scientifiques qui est l'expérimentation. De nombreux équipements de CSTI se sont distingués (certains depuis longtemps comme l'Exploratorium de San Francisco) par cette démarche proposée au public. Certains comme Les Petits Débrouillards en

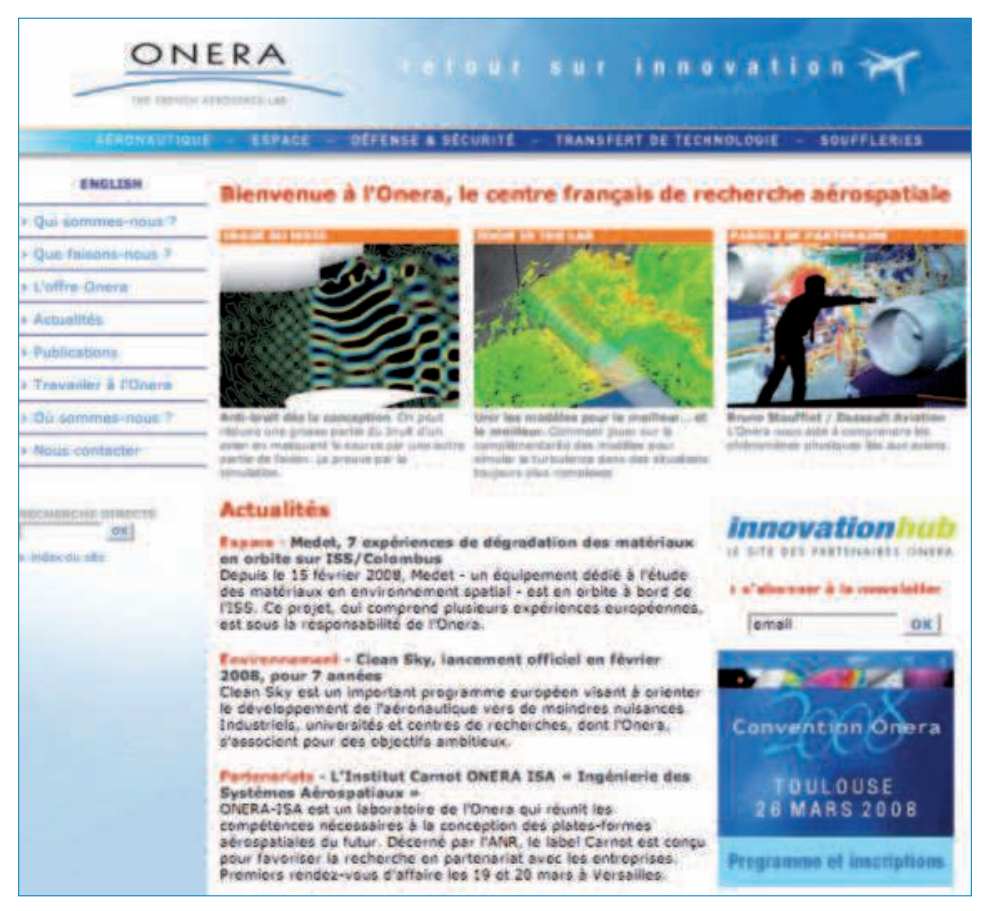

Onera : un site de presse (modèle de la dépêche) avec un compromis de magazine didactique des sciences alliant des images spectaculaires et de longs commentaires. 
font même le cour de leur savoir-faire et cela contribue à les distinguer nettement de nombreuses autres offres, alors que sur leur site Web, cette approche n'est pas si marquée. Le site de la Cité des Sciences et de l'Industrie propose un laboratoire (dont le nom n'est pas si explicite) ainsi que des expériences dans certaines expositions (mais on ne peut pas le savoir sur la page d'accueil), alors que certaines sont remarquablement faites et très originales dans l'offre du Web classique. Le Palais de la Découverte fait aussi une offre d'animations assez simples, mais qui permet de refaire des expériences chez soi et il complète son offre avec des vidéos qui présentent certaines expériences là aussi reproductibles. Une vraie réflexion collective sur cet écart mériterait pourtant d'être mise en place car le Web permet de fournir des outils d'animation interactifs tout aussi performants que ceux qui auparavant étaient réservés aux CD-ROM.

La dimension citoyenne émerge difficilement de l'activité de la CSTI sur le Web. Alors que des débats sur les orientations scientifiques et techniques de nos sociétés se multiplient dans les médias ou dans des régions (pour des équipements particuliers par exemple), la CSTI sur le Web semble peu les relayer (exception notable à Grenoble par exemple) ou, plus important, ne semble pas avoir adopté une politique d'utilisation spécifique du Web pour soutenir ce type de participation. Est-ce à dire que cela révèle la faible place de cette dimension citoyenne dans les pratiques des institutions de la CSTI ? Nous ne pouvons le dire.

Pour terminer, notons que la présentation topologique du Web permet de voir à quel point les grandes institutions de référence extérieures au domaine sont importantes, tout comme la presse.

\section{Résumé des pôles d'attraction dans les stratégies de la CSTI}

Nous avons donc observé certaines des tensions qui structurent le domaine et qui recoupent en partie ce qu'avait repéré Choffel-Mailfert :

- l'ancrage local ou régional et l'attracteur touristique - le rythme des événements proche d'un attracteur médiatique

- la dimension patrimoniale

- la pratique expérimentale

- les enjeux citoyens

- les liens avec les institutions didactiques

Cependant, ces enjeux prennent une autre visibilité à travers l'étude des sites Web et permettent de



Le muséum de Bourges : un site qui propose une recherche aisée des informations

mettre en évidence des stéréotypes dans la politique de culture scientifique qui sont peu adaptés à la médiation demandée par les publics en général, mais plus spécifiquement à ce qui se passe sur le Web avec le Web 2.0. Nous prenons ici le risque de poser les linéaments de ce que pourrait être une politique de « culture scientifique, technique et industrielle 2.0 ».

\section{Conclusion : \\ Pour une stratégie " CSTI 2.0 "}

La CSTI et ses institutions ont certes pris le tournant du Web et sont présentes sur la toile, mais les modèles mis en œuvre reflètent avant tout leurs tensions stratégiques, sans prendre en compte l'autonomie de ce monde. Ce qui était une faiblesse dans le cas du Web originel deviendrait une erreur importante dans le cadre du Web 2.0. Chaque intervenant pour son propre compte peut utiliser ce cadre nouveau du Web 2.0 pour penser une stratégie Web spécifique qui dépasse la seule valorisation des équipements. Et nous avons l'audace de penser que, ce faisant, ils rempliraient de façon plus complète et sans coût très élevé, leur mission de diffusion de la culture scientifique, technique et industrielle.

Le Web 2.0 repose sur des applications qui permettent une collaboration accrue entre internautes : tout internaute peut exploiter des formats de publication simples, tels que les blogs (le succès de Skyblog est un phénomène de société à lui tout seul). Mais certains sites offrent désormais la possibilité de publier tout document rapidement et ce sont les sites à base d'images (Flickr, You Tube, Dailymotion) qui ont le plus de succès, signant ainsi la fin de la domination du Web savant dans les usages. 
Une stratégie CSTI 2.0 s'appuierait sur plusieurs leviers :

- De la culture cultivée à la culture ordinaire. La culture STI n'est pas la propriété des institutions, mais elle est présente dans toutes les activités de la population et le Web 2.0 permet de les valoriser, de les faire circuler.

- Du public consommateur au « proam », l'amateur de sciences éclairé et contributeur. La CSTI 2.0 viserait à encourager et à soutenir toutes les initiatives des internautes, mais aussi des publics à contribuer par leurs documents, leurs données, leurs observations à la constitution des bases de référence.

- De la CSTI officielle à la CSTI débat. Avec le Web 2.0, les outils de débat et de navigation dans les termes d'un débat ou d'une controverse sont disponibles. Dans un tel contexte, les débats ne peuvent être contrôlés, même s'ils peuvent être modérés.

- De la CSTI document à la CSTI expérience. Le travail des scientifiques peut désormais être simulé et les phénomènes éprouvés par l'internaute grâce aux potentiels des lignes ADSL et des logiciels 3D. Mais des expériences superbes peuvent être faites en $2 \mathrm{D}$

- De la CSTI franco-française à la CSTI sans frontières. Les domaines scientifiques et techniques traités aussi bien que l'échelle des débats entre scientifiques sont tous internationaux. Dès lors qu'un site Web français prétend devenir une référence, une autorité ou un hub, dans un domaine précis, il doit étendre son approche au monde entier. - De la CSTI événement à la CSTI capitalisante. De véritables communautés qui pourraient prendre en charge certains des domaines à partir d'un événement donné pour le faire vivre au-delà du moment même d'animation publique et conserver ses traces vivantes.
Notes

(1) Le projet de recherche Web CSTI Culture scientifique et technique sur le Web : mesures des usages et dynamique d'un champ avait été sélectionné dans le cadre de l'appel d'offres « Usages de l'Internet » du ministère de la Recherche, en 2004. Il s'est déroulé sur les années 2005 et 2006 avec la coopération de 4 partenaires fédérés autour de la plate-forme Lutin (Laboratoire des Usages en Technologies d'information numériques, UMS CNRS 2809) qui venait d'ouvrir en septembre 2004 à la Cité des Sciences et de l'Industrie : UTC (Costech), Paris 8 (LCU), Paris 6 (LIP6) et l'AMCSTI. Les compétences réunies étaient donc pluridisciplinaires, informatique, sciences cognitives et ergonomie, sociologie et sémiotique, avec l'appui d'un réseau de partenaires sur le terrain. Le rapport complet est téléchargeable sur le site de l'AMCSTI (www.amscti.fr) et sur celui du Lutin (www.lutin-userlab.fr).

(2) Nous avons déjà mis en œuvre cette méthode pour l'analyse de la lecture sur le Web : Ghitalla, F., Boullier, D. et al. L'outre lecture. Paris BPI, 2004.

(3) C'est notamment le cas pour Aquariums de France qui est un site personnel et qui n'aura pas été étudié dans les trois phases suivant la topologie. Pour la partie des parcours en masse, l'étude n'a pu porter sur la Cité des Sciences et de l'Industrie en raison d'erreurs de transmission de données. De même, les sites du parc du Lubéron, du musée des Arts et Métiers et des Petits Débrouillards n'ont pu être étudiés.

Pour la partie sémiotique, l'étude n'aura porté principalement que sur les sites de la Cité des Sciences et de l'Industrie, du musée des Arts et Métiers, de Cap sciences, et de l'Onera, en raison avant tout de la diversité des traditions sémiotiques mobilisées. Nous avons aussi examiné, mais de façon plus sommaire, l'écomusée du Pays de Rennes, le site des Petits Débrouillards et le CCSTI de Grenoble. Pour l'oculométrie, tous les sites ont été étudiés à l'exception de celui des Aquariums de France. (4) Choffel-Mailfert, M.-J. Une politique culturelle à la rencontre d'un territoire: culture scientifique technique industrielle en région Lorraine. Paris : L'Harmattan, 2000.

(5) Ces travaux ont été réalisés par Mathieu Jacomy et Franck Ghitalla, de l'UTC, dans le cadre du projet Web CSTI. Une entreprise, RTGI, est née depuis à Compiègne qui offre une expertise reconnue sur toutes ces méthodes. 